\title{
Abuso Sexual Infantil desde una perspectiva psicosocial y jurídica
}

\section{Child Sexual Abuse from a psyco-social and legal perspective}

\section{Sexueller Missbrauch con Kindern aus psychosozialer und rechtlicher perspektive}

Tania A Vargas Rubiano ${ }^{1}$, Maribel Mosquera Pastrana', Kelly Yohana Medina Narváez ${ }^{3}$, Miryam C. Fernández Cediel ${ }^{4}$

\section{Resumen}

El abuso sexual infantil (ASI) en la actualidad es una problemática de índole social, que cada vez toma más importancia por el alto impacto psicosocial que tiene sobre los niños del mundo. Es un problema que es necesario tratarlo desde una perspectiva psicológica, jurídica y social donde se toman en cuenta los factores de riesgo tanto para la víctima como para el victimario. El presente artículo expone un contexto general del ASI, su comprensión desde los factores de riesgos que se tienen los niños y niñas. De igual manera se trata de entender los factores de riesgo que hacen que el abusador actué cómo son los trastornos mentales y la fuerte influencia que tiene el ciclo transgeneracional de la violencia. También se expone las consecuencias psicológicas, físicas y sociales que acarrea del ASI, pues es un evento traumático que trae consigo afectaciones en el desarrollo emocional del niño. Se exponen algunas creencias que se tienen con respecto al ASI, el cual se cree que es fruto de la imaginación del niño, que los abusadores pertenecen a un nivel socioeconómico bajo, que todos los abusos son cometidos con violencia. Por último, se entrega una breve exposición sobre lo que significa la intervención psicológica desde una perspectiva integradora (víctima y familia) y Ia intervención psicológica en el campo judicial.

Palabras claves: Abuso sexual infantil, con secuencias psicológicas, físicas, conductuales y sociales, intervención psicológica, intervención jurídica, víctima, victimario.
Abstract

Child sexual abuse (CSA) nowadays is a dilemma of social nature, that each day gains more importance due to its high psychosocial impact that has over the children of the world.

It's an issue that'snecessary to address from a psychological, legal and social perspective where the risk factors are taken into account for both the victim and the aggressor. This article outlinesa general context of the CSA, it's understanding from the risk factors that the boys and girls have. Also it tries to understand the risk factors that make the aggressor to act these mental disorders and the strong influence that the trans-generational cycle has into violence. It's also outlined the psychological, physical and social consequences that the CSA brings, because it's a traumatic event that brings with it damages to the emotional development of the child. Some beliefs about the CSA are outlined, in which some cases the people believe this is part of the child imagination, or the aggressors belong to a low socio-economic level, or that all abuses are made with violence. Lastly, a short description is delivered about what the psychological intervention means from a integrative perspective (victim and family) and the psychological intervention in the legal field.

Keywords: Child sexual abuse, phycologic, physical, behavioral and social consequences, psychological intervention, legal intervention, victim, aggressor.

\section{Zusammenfassung}

Sexueller Missbrauch von Kindern ist in seiner Aktualität ein soziales Problem, welches weiterhin an Bedeutung für den hohen sozialen Einfluss gewinnt, den es auf die Kinder der Welt hat. Es ist ein Problem, das aus einer psychologischen, rechtlichen und sozialen Perspektive betrachtet werden muss, wobei man sowohl die Risikofaktoren für das Opfer, als auch für den Täter berücksichtigen muss.

1 - Miembro del Grupo de Investigación Crecer. Universidad Surcolombiana. Neiva. Colombia. taniavargasru@gmail.com

2 - Miembro del Grupo de Investigación Crecer. Universidad Surcolombiana. Neiva. Colombia. mariamosquera10117@hotmail.com

3 - Miembro del Grupo de Investigación Crecer. Universidad Surcolombiana. Neiva. Colombia. nena_9304@hotmail.com

4 - Magíster en Psicología. Universidad Surcolombiana. Neiva.Colombia. cristina.fernandez@usco.edu.co 
Dieser Artikel stellt einen generellen Kontext des sexuellen Missbrauchs von Kindern zum Verständnis der Risikofaktoren für Jungen und Mädchen dar. Ebenso geht es darum zu verstehen, welche Risikofaktoren den Missbrauchenden zu seinen Handlungen bewegen, welche psychischen Störungen er hat und darum, den starken Einfluss des transgenerationellen Kreislaufs der Gewalt abschätzen zu können. Außerdem stellt er die psychologischen, physischen und sozialen Konsequenzen dar, die durch den sexuellen Missbrauch verursacht werden. Dieser ist ein traumatisches Ereignis, welches zu Beeinträchtigungen in der sozialen Entwicklung des Kindes führt. Der Artikel zeigt auf, dass einige Menschen in Bezug auf den sexuellen Missbrauch glauben, dass er der Vorstellungskraft der Kinder entspringt oder dass die Täter einer niedrigen sozialen Schicht angehören oder dass alle Missbräuche Gewalttaten sind. Schließlich widmet sich ein kurzer Abschnitt der psychologischen Intervention aus der integrativen (Opfer und Familie) und aus der rechtlichen Perspektive.

\section{Contexto General y Factores de Riesgo del Abuso Sexual Infantil}

El Abuso Sexual Infantil (ASI) es un tipo de violencia sexual ejercida por una persona mayor que impone algún tipo de poder hacia un niño o niña. Este acto va desde acciones de contacto visual hasta la penetración, ocasionando daños en las dimensiones psicológicas y físicas e incluso ocasionando la muerte o dejando secuelas a lo largo del desarrollo de la vida. Del mismo modo el ASI también puede ser un hecho realizado por otro menor que tenga mayor edad, o que posea poder o control sobre la víctima (Mebarak,et al. 2010).

Según la Convención de los Derechos del Niño, los niños y niñas deben ser protegidos de cualquier forma de violencia, y por lo tanto también se le deben atender problemas que afectan su integridad y seguridad; sin embargo, ni estos derechos, ni su derecho a ser reconocido su carácter de individuos les son respetados, por lo cual no pueden tomar sus propias decisiones y no son sujetos plenos de derechos (Velázquez, et al. 2013).

En Colombia como en muchos otros países las leyes están para defenderlos y hacer justicia. Lastimosamente a pesar de que los niños y niñas tienen una ley que los defiende (la ley 1098 del 2006), presenta algunas contradicciones que a la hora de hacer justicia, los trabajadores de la ley: abogados, comisarios, defensores de familias y jueces llegan a desconocer. En muchas ocasionales el proceso legal e integral para asistir a los niños y niñas víctima de abuso sexual pueden generar dificultades a la hora de velar por sus derechos de los niños y estos trabajadores de la ley deben entender que en sus manos se encuentra el poder tanto de hacer legítimo el derecho del menor ante lo ocurrido y por otra parte presentar un buen caso y justo que respalde la decisión del fallo contra el abusador (Uribe,2011).

Por otro lado, estos procesos judiciales y el reconocimiento de esta problemática por parte de los académicos ha permitido identificar el perfil del niño o niña abusada, el cual se caracteriza como un ser sacrificado por su familia, ya que si habla esta será destruida, el niño (a) cree estar recibiendo privilegios materiales y afectivos hacia él y su familia por parte de su victimario, por tal razón evita sentirse avergonzado y no quiere poner en riesgo la integridad de su núcleo familiar(Villanueva, 2013).Esta última característica plantea la necesidad de darle mucha credibilidad al testimonio del niño, aprendiendo a entender su lenguaje que se puede basar más en fantasías que en lenguajes de común uso de los adultos (Intebi, 2008). En este último punto, la psicología ha venido trabajando el ASI y en el desarrollo de técnicas e instrumentos que indaguen y suministren un buen diagnóstico para así proporcionar un riguroso tratamiento integrador tanto de la familia como la víctima (Velázquez, et al, 2013).

El abuso sexual infantil presenta una alta prevalencia a nivel mundial, la mayoría de los países tienen y han realizado estudios e investigaciones sobre esta problemática, los datos históricos muestran la alta vulnerabilidad que los niños y niñas han tenido desde siempre en la sociedad (Gómez, Díaz Huertas, \& Casado Flores, 2000).

En Colombia, los factores de riesgo según el Instituto Colombiano de Bienestar Familiar (ICBF) son: ser de sexo femenino, tener entre 11 y 15 años, padecer insuficiencias económicas y culturales, haber sufrido maltratos, tener una baja autoestima, sumisión a la autoridad y poca confianza en los adultos. En efecto un $78 \%$ de los abusadores son conocidos, sean padrastros o familiares y se puede percibir que la mayoría de los abusos son cometidos por varones, esto no quiere decir que las mujeres no cometan abusos, pero son menos implicadas por los estereotipos o roles que ejercen en la sociedad (ICBF, 2007).

Del mismo modo las familias disfuncionales tienden a que sus hijos padezcan abuso sexual, siendo una consecuencia del consumo de drogas, alcohol por parte de sus padres, en la estructura familiar la falta de un modelo paterno y por otro lado la escasez de educación sexual (Mebarak,et al, 2010)

Otro de los factores de riesgo es el hecho de pertenecer a una familia reconstituida, ya que los niños pueden ser abusados por parte de su padrastro o madrastra y la tiranía que ejerce el padre lo hace que exceda de su poder y violente al niño (a). Vemos que aquí el papel de la madre es borrado, ya sea por su ausencia o falta de participación en su hogar y en muchos casos ella acepta el incesto, ya sea porque depende económicamente de su conyugue y busca los mecanismos para comprenderlo y perdonarlo. Estos problemas de inmadurez e irresponsabilidad por parte de la madre, hace que se presenten estos episodios de abuso y a 
veces la falta de una buena actividad sexual por parte de la pareja hace que el hombre busque como satisfacer estos deseos e inmediatamente recurre a buscar lo más cercano para saciarse (Villanueva Sarmiento, et al.2013; Intebi, et al. 2008).

Mebarak, et al, (2010) reconocen cinco factores de riesgo: los familiares, los económicos, la discapacidad física, problemas de desarrollo y variables ambientales como, por ejemplo, la guerra. Este último factor, en el caso colombiano se convierte en un factor de gran preocupación tras mantenernos en un conflicto armado de supera el medio siglo.

\section{Factores de riesgo para que el abusador actúe}

Existen factores de riesgo que hacen que el abusador actué. El primero, debe haber por parte del victimario una motivación que lo lleve a abusar a un niño (a); uno de los elementos problemáticos que puede causar el surgimiento de esta motivación es la experiencia del abuso sexual durante la infancia del abusador, el cual tiene una alta posibilidad de que en ningún momento se haya descubierto y mucho menos denunciado $y$ atendido. Este aspecto plantea la posibilidad de continuar el ciclo transgeneracional de la violencia y el abuso, replicando a otros niños su experiencia traumática. Otra causa de esta motivación es que el abusador puede padecer trastornos o pedofilia, este hecho tiene un gran significado emocional que solo es saciado por un menor. Igualmente se encuentra que el abusador no es capaz de inhibir algunas conductas, es el caso del consumo de alcohol, el cual puede generar un acto de violencia. También se encuentra, en algunos casos, un desentendimiento del estado físico y la incapacidad de reprimir todas estas situaciones que aún le agobian de su infancia (Villanueva et al, 2013; Gómez, Díaz Huertas, \& Casado Flores, 2000).

El agresor es capaz de conocer las barreras que protegen al niño para así vulnerarlas, por ejemplo, la ausencia de la madre, enfermedad, ignorancia frente al tema, falta de vigilancia, hace que se desencadene un episodio de abuso y siendo estos factores los precursores para que el abusador aproveche $\mathrm{y}$ establezca una buena relación de confianza con el niño brindándole el acompañamiento que su familia no le suministra (Villanueva Sarmiento, 2013).

\section{Otras formas de abuso y epidemiología}

El abuso sexual no solo es entendido como el contacto físico sino como algo más amplio donde son considerados como abuso: el manoseo, tocamiento, seducción verbal, masturbación, pornografía, exhibición de genitales, etc. (Mebarak, Martínez, et al, 2010). Es indispensable aclarar que el abuso sexual se presenta también en grupos religiosos, étnicos sin importar la clase social. (Gómez, Díaz Huertas, \& Casado Flores, 2000).Según el Fondo de las Naciones Unidas para la infancia, cualquier niño (a) es susceptible de sufrir un abuso sexual, siendo la edad más prevalente entre los 10 y 14 años, con un 37\%, mientras los niños (as) de 1 a 4 años tienen un $10 \%$, los de 5 a 9 años tienen 25\% y los de 15-17 años tienen un $14 \%$. Todo para un total de $86 \%$ de abusos sexuales en menores de edad entre 13.352 dictámenes de abuso sexual por parte del Instituto Nacional de Medicina Legal y Ciencias Forenses (Mebarak, et al.2010).

Según el ICBF (2007) 11.000 niños y niñas son víctimas de abuso sexual cada año, y esto sin contar que algunos casos no son denunciados por miedo y tampoco son detectados por las familias. Por otro lado, se tiene que contemplar que los abusos sin contacto físico no son denunciados aunque se presenten frecuentemente.

Según Pereda (2009) el 7.9\% de los abusados son hombres y $19.7 \%$ son mujeres, es decir, que las mujeres son más vulnerables y que tienen más factores de riesgos de ser abusadas. En ese sentido, el estudio realizado por los autores en mención revelan que el tocamiento se presenta en un $64.39 \%$, lo cual es muy alto frente a los tipos de abuso con contacto físico que son menos prevalentes, el abuso sin contacto se presenta en un 3.41\% al igual que la masturbación y penetración anal (Mebarak,et al, 2010).

\section{Consecuencias Psicológicas y Sociales en los niños y niñas víctimas de ASI}

El abuso sexual afecta la vida de cualquier persona abusada, su familia y las dimensiones en donde se desenvuelve la víctima (Mebarak, et al, 2010; Villanueva Sarmiento,et al, 2013; Casella, et al, 2013).El ASI es un evento traumático que trae consigo una serie de consecuencias psicológicas que afectan directamente el desarrollo emocional del niño. Las victimas de ASI sufren después del episodio; ansiedad, depresión, vergüenza, disociación, ideación suicida, abusos de sustancias, baja autoestima, baja inhabilidad para el auto cuidado y la sensación de infancia perdida. Todos estos síntomas están relacionados con la culpabilidad que le da la familia o el perpetrador. Las mujeres en la vida adulta pueden desarrollar una angustia sexual frente a su pareja, el stress post-traumático también es un indicador del abuso sexual que deja secuelas.

Del mismo, modo el niño (a) puede presentar trastornos disociativos de la personalidad, donde pierde su identidad, la memoria y hasta la conciencia, todo esto se da porque pretende escapar de la situación para así poder olvidarla.

También se desarrolla un síndrome llamado acomodación sexual que consiste en que el niño (a) se queda callado para proteger a su familia y por las constantes amenazas que recibe por parte del abusador, la desprotección y falta de apoyo que presenta la víctima, son a su vez factores fuertes para el abusador actué llevándolo a que atrape al niño (a) por medio de regalos y demostraciones afectivas las cuales ocasionan que el menor se encuentre en un estado de vulnerabilidad permanente, esto acarrea como consecuencia que el (la) niño (a) se demore en 
denunciar y cuando lo hace no se le cree; porque las autoridades y los padres piensan cómo pudo aguantar tanto tiempo, cómo no decir nada y por lo tanto dudan de su testimonio, en algunos casos la misma madre obliga a que el niño se retracte de lo que dice, solo para que el padre no se vea envuelto en implicaciones legales; finalmente dejan que el niño siga así sin una ayuda para enfrentar este problema que lo agobiara a lo largo de su vida (Villanueva, 2013).

Por otra parte en cuanto los síntomas conductuales, se pueden presentar conductas hiperactivas, problemas de atención y concentración, bajo rendimiento académico, peor funcionamiento cognitivo general, este grupo de síntomas es muy importante, ya que en algunos casos son vistos como conductas propias de los niños sin ser atribuidas a hechos como abuso sexual, esto puede generar nuevamente un círculo vicioso de maltrato a una víctima que ya ha sido vulnerada y que está presentando un fenómeno de causalidad que lo refleja en su conducta(Pereda, 2009).

En cuanto a las relaciones sociales, los niños y niñas pueden presentar problemas de relación: menor cantidad de amigos, menor tiempo de juego con iguales, elevado aislamiento social, lo cual implica deterioró en la calidad de su vida. Aunque las investigaciones muestren los anteriores síntomas como los más influyentes, es de gran importancia tener en cuenta todas las diversificaciones que estos pueden presentar y la forma en la que cada niño (a) puede interactuar, por esto es primordial establecer las variables que pueden estar asociadas con la presencia o ausencia de sintomatología psicológica en víctimas de abuso sexual infantil, teniendo en cuenta el estado individual de cada niño o niña y el contexto sociocultural en el que se desarrolla (Pereda, 2009).

\section{Consecuencias Físicas en los niños y niñas víctimas de ASI}

Otra de las secuelas que se ocasionan por el ASI sonde tipo físico. Pueden verse afectados los niños y niñas en la parte alimentaria, pues pueden presentar problemas de anorexia, ocasionando una gran pérdida de peso y por lo tanto, un retraso en el desarrollo; también presentan amenorreas, problemas de sueño, pérdida de control de esfínteres, del mismo modo se puede presentar lesiones genitales cuando se ha ejercido un alto grado de violencia dentro del abuso; traumatismos e infecciones, y fisuras anales entre otras. Es importante tener en cuenta que los síntomas físicos son altamente variados y en algunas ocasiones son atribuidos a otro tipo de patología, lo cual ocasiona mayor dificultad a la hora de identificar este tipo de abuso; además otra característica de las consecuencias en el área física es que en la mayoría de los casos los síntomas son nulos y tienen la característica de desaparecer rápidamente, en el momento de brindar atención clínica ha trascurrido un periodo prolongado de tiempo por ende la sintomatología puede que haya disminuido y en algunos casos desaparecido(Villanueva, 2013).

\section{Creencias sobre el Abuso Sexual Infantil}

Teniendo en cuenta que el individuo está influenciado por la cultura, vemos como surgen unos mitos respecto al abuso sexual que lo hace minimizar y ocultarse. Entre las creencias se encuentra, por ejemplo, que las historia del abuso sexual infantil son fruto de la imaginación del niño; otra tiene que ver con que los abusadores pertenecen a un nivel socio-económico bajo, lo cual no es cierto ya que existen abusadores con comodidades y que recurren a la prostitución infantil. Por otro lado, tenemos la creencia que todos los abusos son cometidos con violencia; eso no es cierto, ya que los abusadores comienzan a buscar juegos con los niños, empiezan a obsequiarles detalles y todo esto hace que los (as) niños (as), sin saber las verdaderas intenciones de los abusadores, accedan al abuso como algo natural y caricias normales (Acosta, 2004).

Por ser un tema tan complejo en las familias, después de enterados que ocurre el ASI, la mayoría optan por ciertas creencias, prejuicios y justificaciones que evitan responsabilidades y buscan culpables de lo que aconteció. Se cree una barrera de mentiras y engaños las cuales no permiten esclarecer ni brindar apoyo al menor quien en realidad es la verdadera víctima de este delito (Intebi, 2008).

Otra creencia es que las personas piensan que este es un hecho que solo ocurre en la actualidad, solo se lleva a cabo por personas desadaptadas y perversas con fines de dañar la vida del niño (a) inofensivo (a) y a esto agregan que los menores víctimas tal vez hasta se lo buscaron (Intebi, 2008).

Según un estudio hecho por el doctor David Finkelfor, citado en Intebi (2008) realizado a un grupo de estudiantes de secundaria en un colegio de los Estados Unidos, logro descifrar que el 19,2\% de las mujeres y el 8,6\% de los varones entrevistados reconocían haber sido víctimas de situaciones abusivas en la infancia. Esto permite ver, que son datos que lastimosamente la sociedad no conoce y mucho menos los padres; es así que lo que se cree que esto solo ocurre en una menor proporción y en familias disfuncionales, en realidad es un problema que, si pudiéramos entrevistar a muchos menores, las cifras serian sorprendentes en cuanto a la cantidad de niños que han vivenciado cualquier tipo de abuso sexual, esto a pesar de que es un tema difícil de tratar, es un tema real y que se vive con más frecuencia de lo que se cree.

Como ya se ha mencionado, se cree que los responsables de estos actos son las personas pobres y hombres con un tipo de adicción, pero esto no es así, esto es un problema que no importa la raza, la edad, ni mucho menos las clases sociales, desgraciadamente cualquiera lo puede cometer y lo puede sufrir. Pero la idea de que son solo los pobres y las personas adictas es porque así la sociedad nos lo hace ver, los medios de comunicación no se dan por enterados de otros casos de ASI en familias que tienen mejores condiciones económicas, solo brindan información de la población 
de escasos recursos, pero esto no quiere decir que ellos sean los únicos perjudicados de esta dura problemática (Intebi, 2008).

Otro prejuicio que se tiene a la hora de que el (la) niño (a), comenta lo que le está ocurriendo, es que ellos son "fantasiosos" o ese es el concepto que los adultos tienen, y no es del todo erróneo, pues según estudios biológicos si se indican que en cierta edad los niños y niñas experimentan su imaginación y fantasías al máximo en su actuar diario, pero que un menor fantasee y cuente cosas explicitas del acto sexual no es muy "fantasioso".

Finalmente otra creencia sobre el abuso sexual es que este no trae ningún daño en los niños, y es que a pesar de este fenómeno algunos niños continúan comportándose de manera normal y como no presentan anormalidades en su conducta creen que la problemática no causó ningún daño. Esta es la idea superficial que los adultos y padres poseen pero que en realidad no es así, la conducta como tal del menor es provocada por esa capacidad psíquica de defensa que se le conoce como disociación, que a pesar de que vive la peor de las injusticias y abuso, esto no es impedimento para no seguir con su vida intentando pensar en otras cosas que no sea el abuso (Intebi, 2008).

\section{Intervención psicológica desde una perspectiva integradora (Víctima y Familia)}

Teniendo en cuenta que el ASI ha sido una de las formas de maltratos más frecuentes en los últimos tiempos, es de gran importancia tener en cuentalos tipos de tratamientos y la atención psicológica para las víctimas de este fenómeno, estas intervenciones pueden presentar variaciones teniendo en cuenta las características individuales y sociales que cada niño (a) pueda presentar (Echeburúa \& Guerrica echevarría, 2011).

En el diagnóstico psicológico, es necesario que se establezca por medio del rapport. Una buena empatía para que el psicólogo no se muestre como algo amenazante; también se debe establecer un buen diagnóstico a través de entrevistas con los padres y con el niño, con observación y aplicación de pruebas psicológicas, como el test de la figura humana y el test de la casa (Velázquez Velázquez, Degadillo Guzmán, \& González Villanueva, 2013).

Unos de los factores que deben ser tenidos en cuenta en el momento de la intervención son los que tienen que ver con la frecuencia, la gravedad y duración con la que el niño o niña han sido expuestos al AS, además se deben tener en cuenta factores propios de la víctima como sexo, edad, y características emocionales que el niño (a) pueda llegar a presentar (Echeburúa \& Guerrica echevarría, 2011).

Estos aspectos se deben tener en cuenta principalmente porque no todos los niños abusados requieren del mismo tratamiento, es fundamental hacer una evaluación inicial a nivel individual y familiar, con el fin de identificar las características individuales y la sintomatología psicopatológica que el niño esté presentando, igualmente es importante identificar los niños y niñas que han tenido un buen proceso de afrontamiento y de duelo a este hecho para así establecerles factores protectores diferentes, con el fin de no llevar al niño a el fenómeno de re victimización. Una de las estrategias acertadas que el psicólogo puede establecer es crear un plan alternativo e intensivo de orientación con la familia para que esta sea un factor protector para el niño, además se debe establecer una evaluación psicológica periódica para estar atentos ante cualquier situación que el niño o niña pueda presentar (Echeburúa \& Guerrica echevarría, 2011).

Las consecuencias de todos estos abusos son diversas y confunden al niño, llevándolo a tener problemas en el desarrollo de su vida (Acosta Colín, 2004). Por lo tanto, se debe realizar una intervención interdisciplinaria en tres niveles en donde se desenvuelve la víctima: individual, grupal y familiar, por medio de técnicas de entrenamiento, relajación, desensibilización y visualización, con un equipo de trabajo integrativo compuesto por abogados, médicos, psicólogos y trabajadores sociales.

La técnica para una buena atención sin importar porque corriente psicológica se base la intervención debe ser el juego, ya que posibilita al niño la expresión de sus sentimientos sin preocupación o sin consecuencias; por medio de esta técnica se podrá conocer lo que el niño desea hacer, lo que realmente quiere decir y cómo enfrentará el conflicto. (Velázquez Velázquez, Degadillo Guzmán, \& González Villanueva, 2013).

Una vez se tiene identificada la conducta, sentimientos y pensamientos del niño, se pasa a la fase del tratamiento a través de técnicas proyectivas, donde el niño pueda utilizar su imaginación para poder destruir al agresor siendo el niño héroe y el abusador el villano, también se puede hacer por medio de dibujos para la disolución de confusiones, y a través de cuentos y dilemas (Velázquez Velázquez, Degadillo Guzmán, \& González Villanueva, 2013).

Cabe resaltar que la intervención a nivel grupal es muy importante ya que a través de esta el menor puede superar el evento traumático, se incrementa su autoestima, no se ve juzgado y se disminuye la estigmatización; adicionalmente, se mejoran las relaciones humanas y por lo tanto adquiere más confianza en las personas que lo rodean (Velázquez Velázquez, Degadillo Guzmán, \& González Villanueva, 2013).

Dentro de la intervención con la familia es vital tener en cuenta cómo ha sido el afrontamiento ante el hecho que se ha presentado, se deben evaluar si han sido relevantes los relatos del menor o si por el contrario han sido ignorados o se ha culpado al menor de lo 
sucedido, estos factores son considerados ya que son de gran importancia en la aparición o no de la sintomatología que presenta el menor. Esto se realiza con el fin de presentar a los padres y familiares la manera adecuada de afrontamiento y las formas de implementar estrategias y solución de problemas (Echeburúa \& Guerrica echevarría, 2011).

Otro aspecto fundamental es la separación de la víctima de su núcleo familiar, esta separación solo se presenta cuando la familia no proporciona un ambiente protector para el menor y en este caso los encargados son entidades institucionales que proporcionen seguridad integral al menor, en el caso colombiano la institución responsable de estas acciones es el ICBF.

En cuanto a la intervención psicológica con la familia y la víctima se llevan a cabo dos procesos uno educativopreventivo y una segunda intervención clínica. En primera instancia es indispensable presentarle al niño un conjunto de estrategias adecuadas para evitar nuevamente que recaiga en situaciones abusivas, se debe dotar al niño de capacidades emocionales y judiciales para el afrontamiento del hecho abusivo. En cuanto a la intervención psicoeducativa y preventiva el terapeuta debe tener como principal objetivo el establecimiento de conceptos como la sexualidad, teniendo en cuenta la edad y características de la víctima, además debe explicar de forma tranquila al niño o niña que la culpa de lo ocurrido es del agresor, sin importar si el abuso se ha presentado de una forma forzosa o si se ha presentado por engaño (Echeburúa \& Guerrica echevarría, 2011). En cuando a la intervención clínica, se encuentra sujeta a las posturas y enfoques que la psicología clínica plantea.

\section{Intervención Psicológica en el Campo Judicial}

La justicia ha servido para hacer cumplir normas y leyes, las cuales deben ser acatadas por todos los ciudadanos y de lo contrario quien no las cumpla será condenado a multas, trabajos comunitarios e incluso hasta la cárcel. En el caso de Colombia, se encuentra la ley 1098 del 2006 (Código de la infancia y la adolescencia), que van en favor de hacer justicia en todos los ámbitos que tenga que ver con los derechos de niñas y niños, en este caso a las víctimas de abuso sexual infantil.

$\mathrm{Al}$ administrar justicia en Colombia, especialmente en la problemática del ASI, es muy complejo ya que las personas que trabajan en este tipo de casos como abogados y jueces no tienen el suficiente conocimiento sobre el desarrollo psicológico del niño, por lo tanto, a la hora de realizar la entrevista, no tienen en cuenta lo difícil que es para el niño o niña tener que volver a recordar la historia de abuso, ocasionando cierto estrés y re victimización (Uribe, 2011).

En la actualidad, los profesionales de la justicia solo se basan de sugerencias de algunos psicólogos forenses con poca experiencia, los cuales no favorecen en este caso, ya que su ayuda no es la apropiada, por lo que los actores judiciales solo tienen el interés de hacer una entrevista para recolectar información, mas no por velar por el buen estado mental y emocional del niño a la hora de narrar este tipo de historias, es por esto que los verdaderos encargados para entrevistar a un menor como testimonio probatorio son los psicólogos especializados, ya que ellos tienen las herramientas, para poder obtener la información del niño y no generar una re victimización (Uribe, 2011).

En la entrevista se deben realizar preguntas sencillas, que no induzcan a la negatividad, se debe tener en cuenta el número de repeticiones del relato y mirar si coinciden, ya que generalmente el proceso de la denuncia ante la justicia es demorada y durante este lapso de tiempo pueda que el menor cambie su versión por influencia del abusador o familiares o presente un rechazo a colaborar con la exploración, es aquí donde hay que trabajar con el rapport, dinámicas lúdicas y preguntas libres sin pretender que el niño entre en estrés y más bien se permita que ellos se desahoguen y puedan tener al final un alivio de poder contar lo que le sucedió, ya que en muchos otros casos, los niños y adolescentes están sometidos a que lo que dicen no es creíble (Uribe et al, 2011; Rozanski, et al, 2003).

Es debido a estas problemáticas que se dan unas pautas de actuación del psicólogo cuando se presentan estos problemas incluido la ansiedad, la falta de seguridad, la confianza, etc. Por esto es importante entablar seguridad y confianza para que el menor sea libre de contar sus relatos, hacer lo posible para que se sienta a gusto, luego de esto se busca de nuevo volver a aclarar el relato para así llenar ciertos vacíos y por ende sea más completo, luego se busca crear interés al menor para que siga con el proceso de exploración y se sienta a gusto en el desarrollo de las diferentes pruebas psicológicas (Jiménez Cortes \& Martin Alonso, 2006).

Es importante tener en cuenta las narrativas de hechos que plantean los niños para evitar ciertos sesgos o errores que se presenten a la hora de ser juzgado el abusador, y por ende que sea justa su condena, pero algunos de los artículos de la ley de infancia y adolescencia colombiana no les permite a los niños esta libertad de opinión (Uribe, 2011).

No necesariamente los relatos de los niños dan a los psicólogos y a los actores judiciales fe, de que todo lo narrado sea cierto, es por esto, que otras de las problemáticas que se presentan en la administración de justicia es la dificultad en la validación de las pruebas como instrumentos probatorios ante un juez para saber cuáles son los verdaderos problemas psicológicos que presenta tanto el niño como el adulto.

Por último, es importante saber que no solo con indagaciones realizadas por los funcionarios judiciales sirven como evidencia ante el juez, también es necesario la aplicación de pruebas que permitan la evaluación psicológica del niño que ha sido víctima del 
abuso sexual y de test proyectivos que permitan analizar la similitud de estos instrumentos de evaluación para poder determinar de manera detallada y especifica sin necesidad de generalizar. Estos instrumentos son vitales para esclarecer la verdad, ya que el tema de abuso sexual es muy difícil de descifrar por muchas otras variables que pueden o no ayudar a saber la verdad, es difícil pero no imposible, pero con la ayuda de los familiares, profesores, amigos, el propio niño o niña y la ayuda de la justicia y su equipo, se puede llegar a dar veredicto confiable sobre el tema, si se permite la modificación de ciertos artículos en esta ley que por el momento está impidiendo el buen trabajo investigativo de los psicólogos para que con la ayuda de los actores judiciales se lleve a esclarecer todo problema judicial que tenga que ver con el abuso sexual infantil (Uribe, 2011).

\section{Referencias Bibliográficas}

Acosta Colín, N. A. (2004). Y entonces ¿que pasa con la prevención del abuso sexual infantil? Archivos hispanoamericanos de sexología, 81-103.

Casella, A. (2013). Impacto diferencial en abuso sexual infantil segun genero desde un enfoque integrativo supraparadigmático. En Actualizaciones en psicoterapia integrativa (Vol. 5, págs. 82-99). Santiago de Chile.

Echeburúa, E., \& Guerrica echevarría, C. (2011). Pratamiento psicológico de las victimas de abuso sexual infantil intrafamiliar: un enfoque integrador. Psicología conductual, 19(2), 469-486.

Gómez, J. A., Díaz Huertas, J., \& Casado Flores, J. (2000). Atención al abuso sexual infantil, Programa de Atención al Maltrato Infantil Instituto Madrileño del Menor y la Familia Consejería de Servicios Sociales. Madrid.

ICBF, I. c. (2007). Guía de atención para niños, niñas y adolescentes víctimas de violencia sexual. Bogotá: Impresol Ediciones.

Intebi, I. V. (2008). Por donde empezar: juicios y prejuicios. Buenos Aires: Ediciones Granica S.A.

Jimenez Cortes, C., \& Martin Alonso, C. (2006). Valoración del testimonio en abuso sexual (A.S.I). Cuad med forence, 83-102

Mebarak, M. R., Martinez, M. L., Sánchez Herrera, A., \& Lozano, J. E. (enero-junio de 2010). Una revision acerca de la sintomatología del abuso sexual infantil. Psicología desde el caribe (25), 128-154.

Pereda Beltrán , N. (2009). Consecuencias psicológicas iniciales. Papeles del psicólogo, 30(2), 135-144.

Uribe Aramburo, N. I. (2011). Abuso sexual infantil y administración de justicia en Colombia. Pensamiento psicológico, 9(16), 183-202.

Velázquez, (2013). Abuso sexual infantil, técnicas básicas para su atención. Reflexiones, 92(1), 131-139.

Villanueva Sarmiento, I. (2013). El abuso sexual infantil: perfil del abusador, la familia, el niño víctima y consecuencias psíquicas del abuso. Psicogente, 16(30), 451-470. 